DOI: $10.1002 / s m l l .200800565$

\title{
Environmentally Responsive Core/Shell Particles via Electrohydrodynamic Co-jetting of Fully Miscible Polymer Solutions
}

\author{
Abbass Kazemi and Joerg Lahann*
}

\begin{abstract}
$\boldsymbol{H}_{\text {erein it is demonstrated that electrohydrodynamic co-jetting is not limited }}$ to Janus-type particles, but can also be used for the preparation of core/shell particles. Using side-by-side flow of miscible polymer solutions, electrohydrodynamic co-jetting offers an elegant and scalable route towards preparation of core/shell particles with otherwise difficult-to-prepare particle architectures, including particles with hydrophilic shell and core. Throughout this study, electrohydrodynamic co-jetting of aqueous solutions consisting of a mixture of PAAm-co-AA and PAA is used, and a range of different types of particles with distinct compartments are observed. Transition from Janus particles to core/shell particles appears to be caused by changes in the relative conductivity of the two jetting solutions. After crosslinking, the core/shell particles are stable in aqueous solution and exhibit reproducible swelling behavior while maintaining the original core/ shell geometry. In addition, the $\mathrm{pH}$-responsiveness of the particles is demonstrated by repeatedly switching the environmental pH between 1.3 and 12. Moreover, the core/shell particles show surprising uptake selectivity. For instance, a 450\% increase in uptake of 6-carboxyfluorescein over rhodamine $B$ base is found.
\end{abstract}

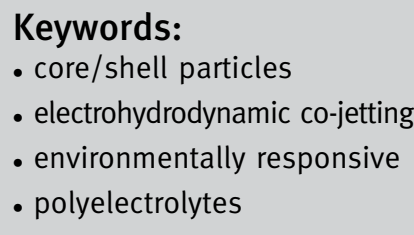

Keywords:

- core/shell particles

- electrohydrodynamic co-jetting

- environmentally responsive

\section{Introduction}

Particles with diameters between $100 \mathrm{~nm}$ and a few micrometers are of immense interest because of their potential applications as drug-delivery systems, ${ }^{[1-5]}$ sensors, ${ }^{[6-7]}$ microreactors, ${ }^{[8]}$ in combined imaging and therapeutics (theranostics), ${ }^{[9]}$ or as food-packaging materials. ${ }^{[10]}$ In particular, environmentally responsive particles, such as $\mathrm{pH}$-responsive core/shell particles, have been under intense

[*] Prof. J. Lahann, Dr. A. Kazemi ${ }^{[+]}$

Departments of Chemical Engineering and

Materials Science and Engineering

Macromolecular Science and Engineering Center

The University of Michigan

48109 Ann Arbor, MI (USA)

E-mail: lahann@umich.edu

[+] Present address: Division of Polymer Science \& Technology Research Institute of Petroleum Industry

P.O.Box 14665-1998, Tehran (Iran) investigation for their ability to produce custom-tailored release profiles. ${ }^{[1,12]}$ A series of methods to prepare core/shell particles has been already established, including emulsion polymerization, ${ }^{[13-16]}$ layer-by-layer (LbL) adsorption onto solid particles, ${ }^{[17-20]}$ templated polymerization, ${ }^{[21-23]}$ and template-assisted electropolymerization. ${ }^{[23]}$ Using a LbL approach, pH-sensitive core/shell particles, as well as hollow capsules, have been prepared. ${ }^{[19,20]}$ Another approach to prepare $\mathrm{pH}$-sensitive core/shell particles is the assembly of macromolecules that display $\mathrm{pH}$-responsive properties ${ }^{[24-26]}$ and undergo biodegradation. ${ }^{[27]}$ In related work, electrospinning has been employed to prepare core/shell nanofibers by co-jetting of two non-miscible solutions in a coaxial-needle configuration. ${ }^{\text {[28-34] }}$

We have recently used electrohydrodynamic co-jetting to prepare biphasic $^{[35]}$ and triphasic nanocolloids ${ }^{[36]}$ (i.e., solid particles with multiple compartments). This approach towards designer nanocarriers is unique in that it enables design of particles with multiple, distinct surface patterns or nanocompartments. Because of its intrinsic simplicity and versa- 
tility, the electrohydrodynamic co-jetting process can be applied to a wide range of specialty and non-specialty materials. The fact that each compartment can be designed independently of the other compartments allows combination of multiple, essential materials functions. Using a side-by-side arrangement of inlet flows to introduce multiple polymer solutions into an electric field, we demonstrated that bi- and triphasic particles can be obtained by simple co-jetting of aqueous solutions containing poly(acrylamide-co-acrylic acid) (PAAm-co-AA) and poly(acrylic acid) (PAA). Moreover, these particles can be stabilized via thermal crosslinking ${ }^{[37]}$ and be selectively surface modified for each compartment. ${ }^{[35]}$ Biphasic nanoparticles showed satisfying biocompatibility in short-term cytotoxicity studies. ${ }^{[38]}$ In contrast to many of the existing methods of particle fabrication such as emulsion polymerization, this approach does not require use of immiscible solutions.

Herein, we demonstrate that electrohydrodynamic co-jetting is not limited to Janus-type particles, but can also be used for preparation of core/shell particles. Using side-byside flow of miscible polymer solutions, electrohydrodynamic co-jetting offers an elegant and scalable route towards preparation of core/shell particles with otherwise difficultto-prepare particle architectures, including particles with hydrophilic shell and core. Throughout this study, electrohydrodynamic co-jetting of aqueous solutions, consisting of a mixture of PAAm-co-AA and PAA is used and a range of different types of particles with distinct compartments is observed. Transition from Janus particles to core/shell particles appears to be caused by changes in the relative conductivity of the two jetting solutions.

\section{Results}

Experimentally, electrohydrodynamic co-jetting relies on parallel extrusion of two miscible solvents through a macroscopic nozzle with a diameter of $0.46 \mathrm{~mm}$. A counterelectrode, which acts as the collection reservoir, is placed about $33 \mathrm{~cm}$ away from the tip of the nozzle. The polymer solution is then accelerated towards the collection electrode by application of an electrical potential of approximately $16 \mathrm{kV}$, which corresponds to an electric field of about $48500 \mathrm{~V} \mathrm{~m}^{-1}$. Due to acceleration in the electric field, the polymer thread is rapidly elongated resulting in a reduction in diameter by several orders of magnitude. This reduction in thread diameter is accommodated by a dramatic increase in surface area, which in turn results in instantaneous evaporation of the solvent, and solidification of polymers and other additives that were initially dissolved in the solvent. Because the solidification process is faster than competing transport/mixing processes between the individual solutions, the original droplet geometry is maintained during the co-jetting, resulting in solid particles with defined compartments. Electrohydrodynamic jetting is a rather complex process, and the ultimate particle geometry depends on a variety of parameters, which may be classified as material- or process-related factors. Critical material-related parameters include viscosity, conductivity, or free surface energy of the jetting solutions, while process-related parameters include the magnitude of the applied electric field, solution flow rates, nozzle geometry, and temperature. In the case of the set-up used for electrohydrodynamic co-jetting in this study, the materials-related parameters can be independently controlled for each polymer solution, while most of the process-related factors cannot be controlled independently.

Figure 1 illustrates the formation of the core/shell particles by electrohydrodynamic co-jetting. In order to be able to visualize the core/shell character of the particles, tetramethylrhodamine isothiocyanate- (TRITC) or fluorescein isothiocyanate (FITC)-dextran were added to the polymer solutions. The polymer solutions used for the jetting process were PAAm-co-AA ( $5 \%$ w/v), PAA $(0.9 \%$ w/v), TRITC-dextran $(0.4 \% \mathrm{w} / \mathrm{v})$ and PAA $(13.1 \% \mathrm{w} / \mathrm{v})$, PAAm-co-AA $(0.044 \% \mathrm{w} / \mathrm{v})$, $\mathrm{NaOH}(2.19 \% \mathrm{w} / \mathrm{v})$, and FITC-dextran $(0.4 \% \mathrm{w} / \mathrm{v})$. Phases containing FITC and TRITC form the particle core or shell, indicated by green fluorescence or red fluorescence, respectively.

As summarized in Table 1, the base polymers of PAA and PAAm-co-AA were used for all solutions in our electrohydrodynamic co-jetting experiments. Addition of $\mathrm{NaOH}$ alters the conductivity of the jetting solution, and the resultant particle architectures range from Janus particles to core/shell particles. Characteristic examples are shown in Figure 2. In the case of the Janus particles (i.e., (biphasic) $)_{\mathrm{a}^{\prime} \mathrm{b}}$ particles; Figure 2a), the ratio of PAA and PAAm-co-AA was identical in both phases. It is important to note that the conductivity of both jetting solutions $\left[\mathrm{a}^{\prime}\right]$ and $[\mathrm{b}]$ was the same $\left(5 \mathrm{mS} \mathrm{cm}^{-1}\right)$. In contrast, simply increasing the PAA ratio of one jetting solution resulted in transition from Janus-type particles to well-defined core/shell particles (core/shell) $)_{\mathrm{ab}}$ particles (Figure 2d). In the case of (core/shell) $)_{\mathrm{ab}}$ particles, addition of $\mathrm{NaOH}$ to only one jetting solution resulted in a conductivity mismatch, where the conductivity of solution [a] was about 6 times higher than that of solution [b]. These relatively small compositional differences have a profound impact on the jetting behavior and the resulting particle geometries.

The difference in the resultant particle geometry is thought to be due to the disparity in the polymer solution conductivities. Electrohydrodynamic jetting relies on the

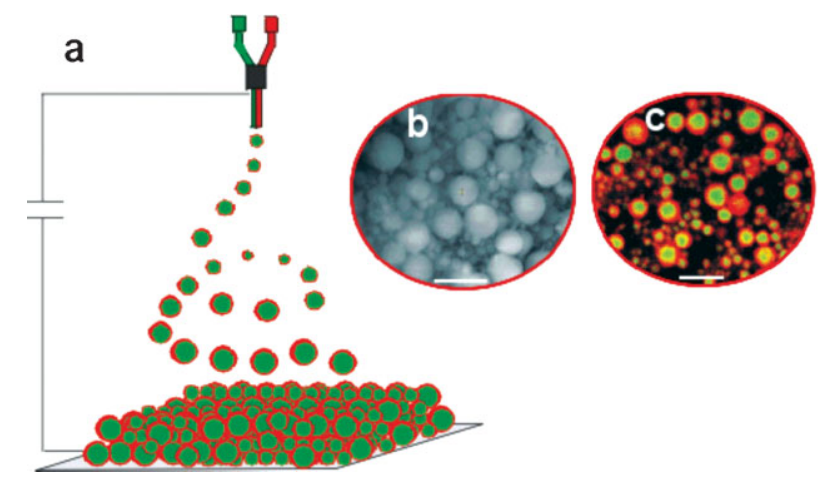

Figure 1. a) Schematic illustration of the electrified-jetting set-up, b) SEM image of microspheres after jetting, c) confocal microscopy images of the microspheres after jetting, indicating the core/shell structure with an average size distribution of $1.9 \pm 0.4 \mu \mathrm{m}$. All scale bars represent $8 \mu \mathrm{m}$. 
Table 1. Composition of polymer solutions used for preparation of (core/shell) $)_{a b}$ or (biphasic) ${ }_{a^{\prime} b}$ particles and particles with undefined geometry ([cb] particles or [db] particles).

\begin{tabular}{|c|c|c|c|c|c|}
\hline & \multicolumn{5}{|c|}{ jetting solutions } \\
\hline & a & $\mathrm{b}$ & c & $d$ & $a^{\prime}$ \\
\hline \multicolumn{6}{|c|}{ polymer solution compositions [w/v \%] } \\
\hline PAA & 13.1 & 0.9 & 13.1 & 13.1 & 0.9 \\
\hline PAAm-co-AA & 0.044 & 5 & 0.044 & 0.044 & 5 \\
\hline $\mathrm{NaOH}$ & 2.19 & - & 0.75 & 1.75 & - \\
\hline dextran & FITC: 0.4 & TRITC: 0.4 & FITC: 0.4 & FITC: 0.4 & FITC: 0.4 \\
\hline conductivity $\left[\mathrm{mS} \mathrm{cm}^{-1}\right]$ & 32 & 5 & 12 & 27 & 5 \\
\hline
\end{tabular}

[a]/[b]: used for preparation of the particles with core/shell geometry.

$\left[a^{\prime}\right] /[b]$ : used for preparation of the particles with Janus geometry.

$[c] /[b]$ or $[d] /[b]$ : same polymer concentrations as $[a] /[b]$, but with different $\mathrm{NaOH}$ concentration, resulting in changes in jetting solution conductivity.

creation of surface charges during the acceleration of polymer solutions in the high electrical field between the conductive needle and the counterelectrode. In principle, the electrohydrodynamic co-jetting process is compatible with a range of different polymer solutions, including aqueous and organic solutions, which can be associated with a range of different conductivities. If polymer solutions with significantly different conductivities are co-jetted, each phase of the droplet accelerates unevenly towards the counterelectrode. Solutions with lower conductivity typically yield higher jet velocities due to higher interfacial charge build-up. In a simplified model of electrohydrodynamic co-jetting of two solutions with distinct conductivities, the solution with lower conductivity functions as the lead phase. The lead phase is initially accelerated, but immediately creates a viscous drag on the second solution, which is pulled along and may be considered a dependent phase. In Figure 2d, the less conductive lead phase is indicated in red, forming the shell of the resultant particles, while the dependent phase tends to form the core. The degree to which a concentric geometry is reached depends on the relative conductivities of the two phases (Figures $2 b$ and $c$ ). As the

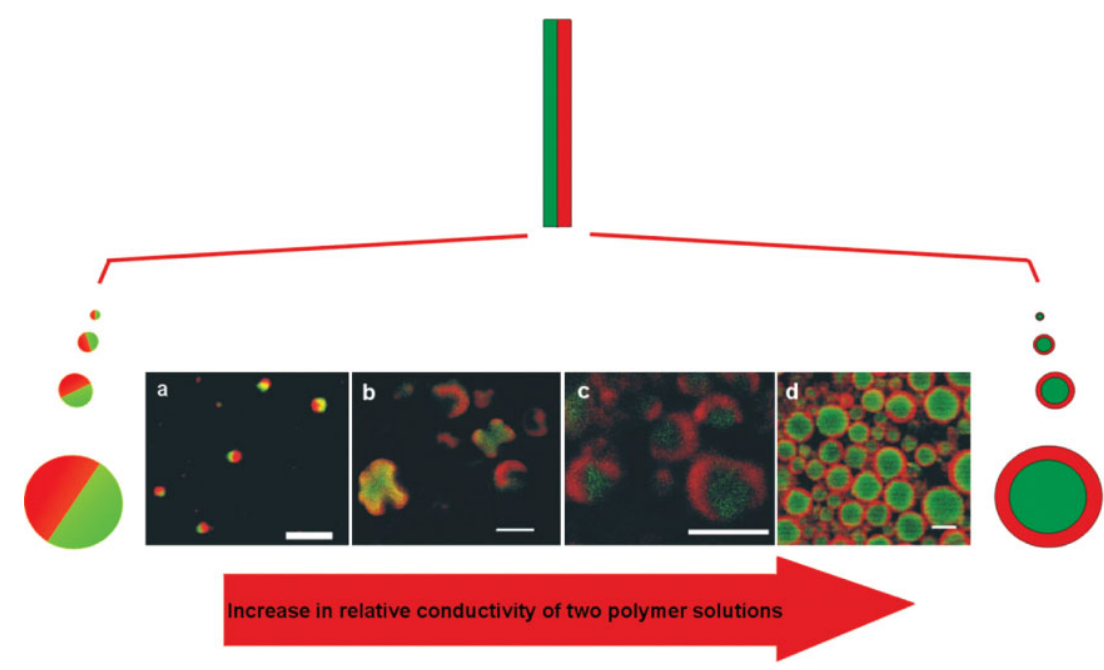

Figure 2. From (biphasic) $)_{a^{\prime} b}$ to (core/shell $)_{a b}$ particles by using side-by-side co-jetting and changing the conductivity of the jetting solution in one of the phases. a) (Biphasic) $)_{a^{\prime} b}$ particles, and b) (core/shell) $)_{a b}$ particles imaged in de-ionized water. Scale bars: $8 \mu \mathrm{m}$. differences in conductivity change from 6- to 3 - to 1.3 -fold difference, the resulting particles lose their core/shell character and begin to resemble Janus particles. While core/ shell particles have previously been fabricated using a coaxial needle geometry with one needle embedded in the second one, our work shows that electrohydrodynamic co-jetting using a side-by-side arrangement of needles can result in both Janusand core/shell particles. Moreover, the transition between the two characteristic morphologies can be controlled by simply altering the relative conductivities of the jetting solutions.

Prior to suspension in aqueous buffer systems, thermal crosslinking between amide and carboxylic groups was necessary to prevent dissolution of the particles in aqueous solutions and to provide differential swelling properties. Polymer crosslinking was performed by placing the particles in an oven at $170{ }^{\circ} \mathrm{C}$ for $2 \mathrm{~h} .{ }^{[37]}$ Under these conditions, the shell phase was more extensively crosslinked due to the higher content of acrylamide in the shell as compared to the core. Particles show reproducible changes in swelling after incubation in aqueous solutions that were either acidic $(\mathrm{pH} 1.3)$ or basic ( $\mathrm{pH}$ 12). Under these conditions, the gross morphology of the particles remained unaltered. Average particle diameters were measured using confocal microscopy and mean values for different conditions are presented in Figure 3.

During electrohydrodynamic co-jetting, a typical batch of particles had an average diameter of $1.9 \pm 0.4 \mu \mathrm{m}$. After subsequent incubation in de-ionized water, the average size of the same batch of particles increased to $6 \pm 1.0 \mu \mathrm{m}$, an increase of more than $300 \%$ in particle diameter. Next, particles were alternately incubated in acidic $(\mathrm{pH}$ 1.3) or basic ( $\mathrm{pH} 12)$ solutions. Under these conditions, the particle diameter reproducibly switched between $3.5 \pm 0.5 \mu \mathrm{m}$ and $6 \pm 1.0 \mu \mathrm{m}$. After 5 consecutive switching cycles the particles maintained their integrity and preserved the core/shell character, suggesting excellent structural stability. The increase in size of the particles in water or basic solutions is due to $\mathrm{pH}$ - 
induced swelling of polyacrylates. Prior to exposure of freshly jetted and cross-linked particles to water, PAA segments form coiled chains. Subsequent exposure to water causes hydration of the particles and results in repulsive interactions between negatively charged carboxylic acid groups. Under these conditions the polyelectrolyte chains exhibit a more stretched form, and inter- and intramolecular repulsion causes increased absorption of water and expansion of the particles. When exposed to an acidic media, the carboxylicacid groups are protonated, eliminating charge-charge repulsion and cause shrinkage of the particles. ${ }^{[39,40]}$ In Figure 3, histograms are shown for each condition indicating the number of particles that fall within one of four size ranges; smallest ( $<25 \%)$; small/medium (26-50\%); medium/large (51-75\%); and largest particle fraction $(>75 \%)$. When exposed to a sufficiently high $\mathrm{pH}$, the acid groups are deprotonated and repulsion between negatively charged polymer chains causes expansion of the particles.

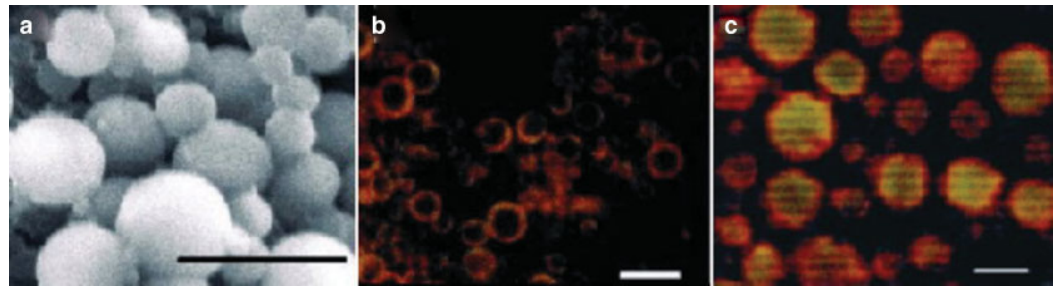

Figure 4. a) SEM image of the particles, which were introduced in an acidic solution $(\mathrm{pH} 0.4)$ and dried in air. b) Confocal microscopy image of (core/shell) ab particles in acidic media at $\mathrm{pH}$ 0.4. c) Confocal microscopy image of the particles after increasing the $\mathrm{pH}$ to 12 by adding $\mathrm{NaOH}$ solution to the media originally containing the particles at $\mathrm{pH} 0.4$. All of the scale bars represent $8 \mu \mathrm{m}$.

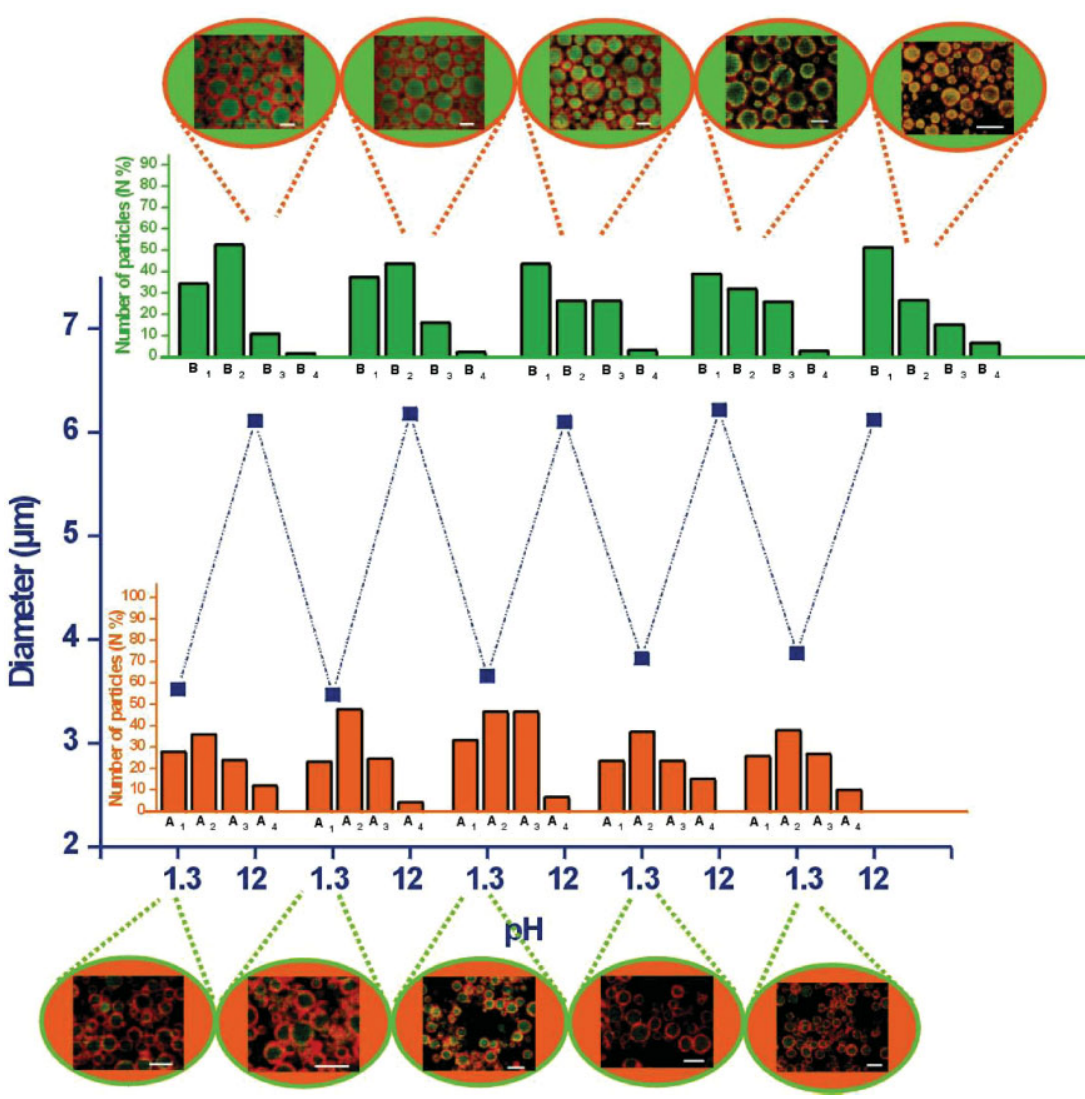

Figure 3. Representation of the swelling behavior of (core/shell) $)_{a b}$ particles when introduced to media at $\mathrm{pH} 1.3$ or 12 . The $\mathrm{pH}$ of the solutions were changed using $\mathrm{HCl}$ or $\mathrm{NaOH}$. All scale bars: $8 \mu \mathrm{m}$. Histograms indicate statistic calculations for size distribution of (core/shell) $)_{\mathrm{ab}}$ particles in acidic $\left(A_{\text {index }}\right)$ or basic $\left(B_{\text {index }}\right)$ environment in a given diameter range; (core/ shell $)_{a b}$ particles at $\mathrm{pH}$ 1.3: 0.6-2.5 $\mu \mathrm{m}\left(\mathrm{A}_{1}\right), 2.5-4.4 \mu \mathrm{m}\left(\mathrm{A}_{2}\right), 4.4-6.3 \mu \mathrm{m}\left(\mathrm{A}_{3}\right), 6.3-8.2 \mu \mathrm{m}$ $\left(A_{4}\right)$; (core/shell $)_{a b}$ particles at $\mathrm{pH} 12: 0.6-4.6 \mu \mathrm{m}\left(B_{1}\right), 4.6-8.6 \mu \mathrm{m}\left(B_{2}\right), 8.6-12.6 \mu \mathrm{m}\left(B_{3}\right)$, 12.6-16.6 $\mu \mathrm{m}\left(\mathrm{B}_{4}\right)$.
Figure 4 shows SEM and confocal microscopy images of $(\text { core/shell })_{a b}$ particles. Figure $4 \mathrm{a}$ shows representative particles which were placed in strongly acidic solution ( $\mathrm{pH} 0.4$ ) and dried in air, indicating that the surface of the particles remains unaltered after introducing them to the acidic medium. However, the confocal microscopy image (Figure 4b) from the particles in acidic media ( $\mathrm{pH} 0.4$ ), suggests that particles became hollow when placed in strong acid. A possible explanation for the transition into hollow capsules is that PAA, which is the dominant polymer in the core, becomes fully protonated leading to a retraction of the polymer chains into the core/shell interface. Dissolution of the core polymer or dye can be excluded as a possible explanation, as particles fully recovered their original core/shell structure after incubation in basic ( $\mathrm{pH}$ 12) solution (Figure 4c). However, mixing of dyes may occur to some extent, as indicated by the color change of the cores from the original green to a more yellowish appearance.

The ability to switch between capsules and core/shell particles may be useful for drug delivery applications or selective absorption of small molecules, as well as environmentally friendly applications, such as remediation of toxins or contaminants.

Figure 5 shows digital photos of a polymer scaffold consisting of (core/shell) $)_{\mathrm{ab}}$ particles, after jetting, in de-ionized water and in acidic media. Immediately upon placing (core/shell) $)_{\mathrm{ab}}$ particles in water (Figure 5b), the particles expand their size resulting in swelling of the scaffold. After altering the environment to an acidic media, the swelling of the particles is reversed with significant shrinkage (Figure 5c).

Next, we evaluated the ability of core/ shell particles to selectively absorb different dyes. Initially (core/shell) $)_{\mathrm{ab}}$ particles with colorless cores were prepared. For better imaging, the shell was either loaded with TRITC- or FITC-dextran. First, the FITC- 


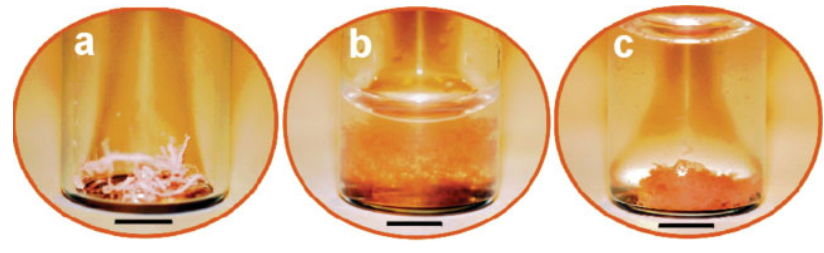

Figure 5. Vials containing (core/shell) $)_{\mathrm{ab}}$ particles after jetting a) in air, b) in de-ionized water, $\mathrm{c}$ ) in acidic media (pH 0.4). All scale bars: $5 \mathrm{~mm}$.

loaded particles were suspended in water (Figure 6a) and a dilute solution of rhodamine $\mathrm{B}$ base was added to the particle suspension. After incubation for $3 \mathrm{~h}$, the red dye was readily taken up into the core of the particles (Figure 6b); enhanced red fluorescence was observed in the shell as well as the core, while the initial dye solution appeared to be depleted. In stark contrast, (core/shell) ab particles without FITC-dextran did not show any appreciable uptake of 6-carboxyfluorescein as shown in Figure 6d for particles with TRITC-labeled shells.

We attribute the striking selectivity of (core/shell) ab particles for different dyes (Figure 7) to electrostatic repulsion due to differences in the net charge of the two dyes. The core/shell particles are net negatively-charged and repel the negativelycharged6-carboxyfluorescein. Tofurther elucidate the molecular basis of this remarkable selectivity between dyes of similar size, we conducted quantitative uptake studies with 6 dyes (Figure 7). The dyes selected for these experiments had molecular weights ranging from 204 to $643 \mathrm{~g} \mathrm{~mol}^{-1}$, andincluded widely used fluorescence dyes, such as rhodamine B, 6-carboxyfluorescein, eosin, and aminoacridone. First, we dissolved pairs of low molecular-weight dyes in aqueous solutions and then added a defined amount of (core/shell) $)_{\mathrm{ab}}$ particles to the solution and incubated for $3 \mathrm{~h}$. Here, neither core nor shell was loaded with any dye during electrohydrodynamic co-jetting. The particles were separated by centrifugation and the supernatant was analyzed by UV/vis spectroscopy. We defined selectivity as the ratio of the two dye concentrations after particle exposure, each being normalized to the initial dye concentration:

$S=\frac{C_{1} / C_{1}^{\prime}}{C_{2} / C_{2}^{\prime}}$

$S=$ dye selectivity; $C_{1}$ and $C_{2}=$ dye concentration after incubation with particles; $C^{\prime}{ }_{1}$ and $C_{2}^{\prime}=$ initial dye concentrations

The previously studied rhodamine $\mathrm{B}$ base/6-carboxyfluorescein pair (i.e., the pair shown in Figure 6) exhibited the highest selectivity with $\mathrm{S}=4.5$. The inset of Figure 7 shows the dye solution before and after incubation with particles. A clear change in color due to selective removal of rhodamine $\mathrm{B}$ base $(\mathrm{RBB})$ can be observed. In contrast, the initially colorless particles are indicated with a bright red color due to selective uptake of only the red dye. A comparable selectivity was achieved for a solutions containing rhodamine $\mathrm{B}$ base and either eosin yellowish solution (EYS) or 3-acetylumbelliferon (AU). When combined with 6-carboxyfluorescein (CFI), the uptake selectivity of rhodamine B (RB) was 2.3, which is significantly lower than the uptake selectivity of rhodamine B base relative to CFI. Moreover, the uptake of ACC was 2.5-fold higher than the uptake for AU, in spite of the fact that AU is the smaller molecule. However, the selectivity of ACC versus AU is significantly lower than the relative selectivity of RB versus AU. Again, this effect does not correlate with molecular weight because the molecular weight of RB is higher than the molecular weight of ACC or AU. Based on these outcomes, several common trends can be identified: i) the presence of carboxylic acid groups in a molecule reduces uptake into particles, presumably due to charge repulsion; ii) amino groups enhance dye uptake - with tertiary amines showing higher uptake than primary amines; iii) presence of a free acid group in $\mathrm{RB}$ decreases uptake and reduces selectivity as compared to $\mathrm{RBB}$; and iv) within the limited number of molecules tested, the process appears to be independent of the molecular weight of the molecules. Future work is needed to further elucidate the underlying mechanism and the boundaries of this striking selectivity.
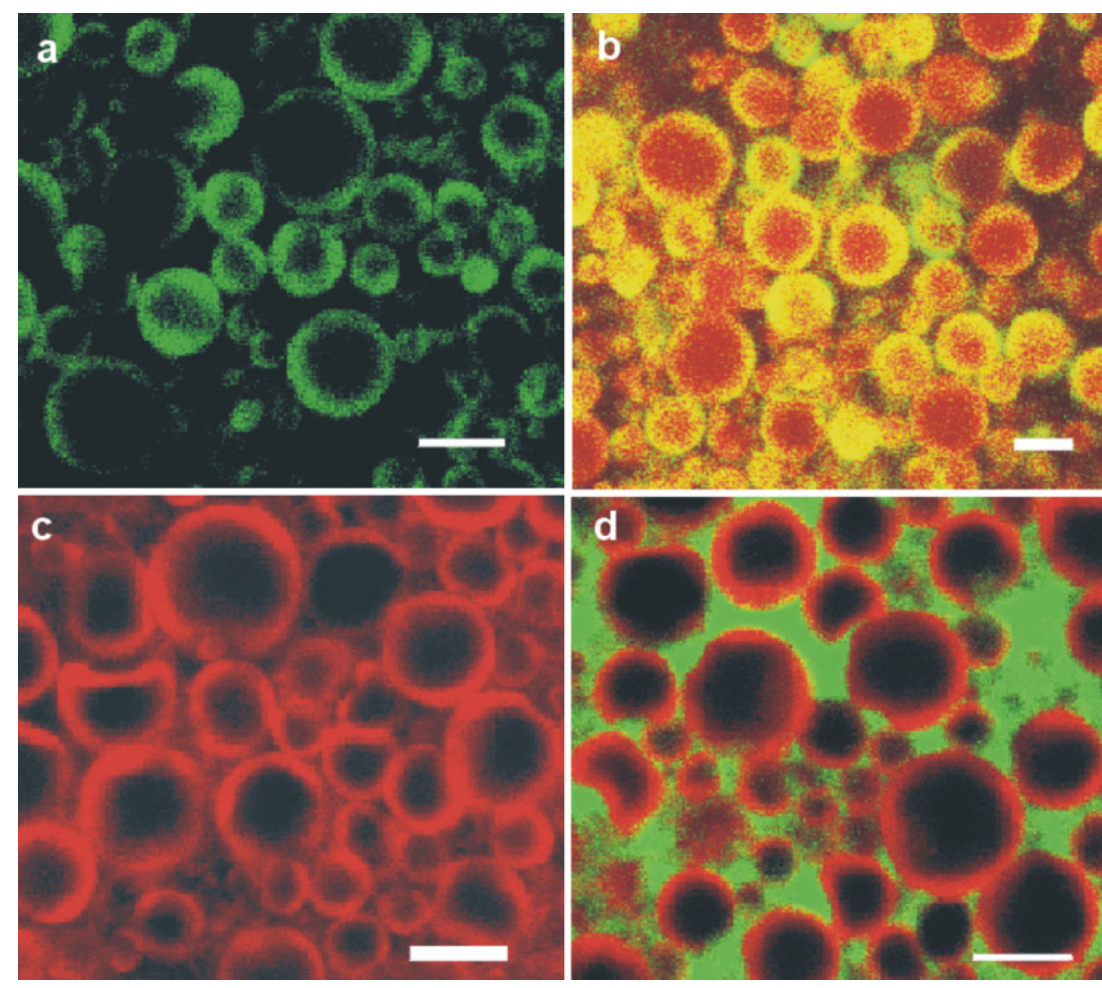

Figure 6. Confocal microscopy images; a) (core/shell) a,b $_{\text {b }}$ particles in de-ionized water (shell: PAAm-co-AA $\left(5 \% w^{-1}\right)$, PAA $\left(0.9 \% w^{-1}\right)$, FITC-dextran $\left(0.4 \% w v^{-1}\right)$, core: PAA $(13.1 \%$ $\left.w v^{-1}\right)$, PAAm-co-AA $\left(0.044 \% w^{-1}\right), \mathrm{NaOH}\left(2.19 \% \mathrm{w} \mathrm{v}^{-1}\right)$ ). b) (core/shell) ${ }_{\mathrm{a}, \mathrm{b}}$ particles (as in Figure $6 \mathrm{a}$ ) in dilute solution of rhodamine $\mathrm{B}$ base. c) (core/shell $)_{a, b}$ particles in de-ionized water (shell: PAAm-Co-AA $\left(5 \% w^{-1}\right)$, PAA $\left(0.9 \% w^{-1}\right) /$ TRITC $\left(0.4 \% w^{-1}\right)$, core: PAA $\left(13.1 \% w v^{-1}\right)$, PAAm-co-AA $\left.\left(0.044 \% w^{-1}\right), \mathrm{NaOH}\left(2.19 \% w v^{-1}\right)\right)$. d) (core/shell) ${ }_{a, b}$ particles (as in Figure $6 \mathrm{c}$ ), in a dilute solution of 6-carboxyfluorescein. All scale bars: $8 \mu \mathrm{m}$. 

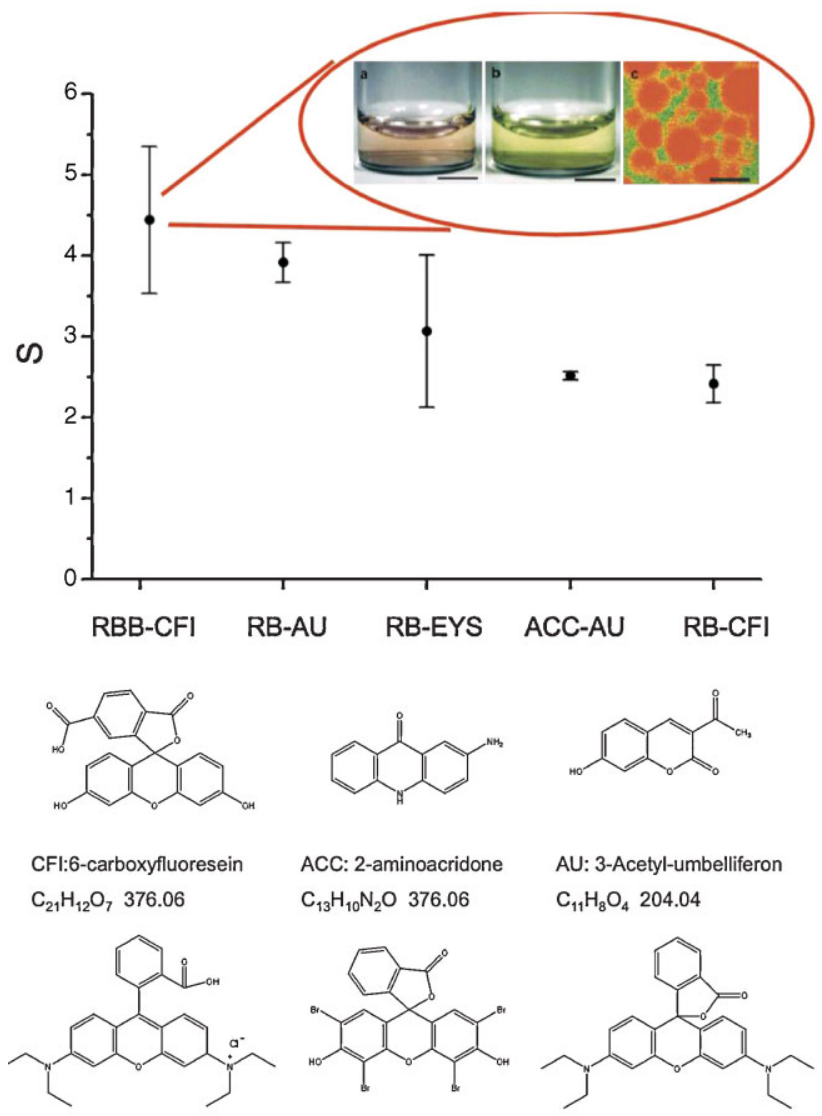

AU: 3-Acetyl-umbelliferon $\mathrm{C}_{13} \mathrm{H}_{10} \mathrm{~N}_{2} \mathrm{O} 376.06$ $\mathrm{C}_{11} \mathrm{H}_{8} \mathrm{O}_{4} 204.04$
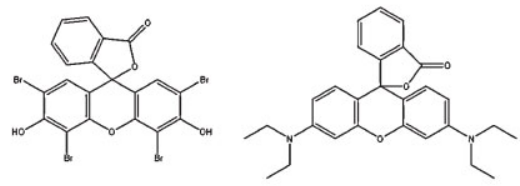

RB: Rhodamine B $\mathrm{C}_{28} \mathrm{H}_{32} \mathrm{CIN}_{2} \mathrm{O}_{3} 479.21$

EYS: Eosin yellowish solution $\mathrm{C}_{20} \mathrm{H}_{8} \mathrm{Br}_{4} \mathrm{O}_{5} 643.71$

RBB: Rhodamine B Base $\mathrm{C}_{28} \mathrm{H}_{30} \mathrm{~N}_{2} \mathrm{O}_{3} \quad 442.23$

Figure 7. Selective uptake of different dyes. Each data point represents the $n$-fold increase in dye uptake after adding core/shell particles to the solution. a) Solution of rhodamine B base and 6-carboxyfluorescein before adding (core/shell) a,b $_{\text {b }}$ particles. b) Solution (as in Figure 7a) upon addition followed by removal of the (core/shell) $)_{a, b}$ particles. c) Confocal microscopy image of (core/shell $)_{a, b}$ particles after being exposed to a solution of rhodamine B base (Figure 7a) and 6-carboxyfluorescein (Figure $7 b$ ). The particle core was initially made colorless. Scale bars for (a) and (b): $5 \mathrm{~mm}$, scale bar for (c): $8 \mu \mathrm{m}$.

\section{Conclusions}

In summary, this work provides a method for preparation of core/shell particles with well-defined structures using electrohydrodynamic co-jetting of two aqueous polymer solutions, containing poly(acrylic acid) and poly(acrylamide$c o$-acrylic acid). Controlled variation of relative conductivities of the two jetting solutions resulted in polymer particles with either Janus-type or core/shell geometries for the same polymer system. After cross-linking, core/shell particles were stable in aqueous solutions, and exhibited reproducible swelling behavior, while maintaining the original core/shell geometry. In addition, $\mathrm{pH}$-responsiveness of the particles was demonstrated by repeatedly switching the environmental $\mathrm{pH}$ between 1.3 and 12 . Moreover, core/shell particles show $450 \%$ increase in uptake of 6-carboxyfluorescein over rhodamine B base; in fact, this appears to be a general trend, as similar selectivities were observed for a number of different dyes. Differential uptake of small molecules and environmental

responsiveness are key features when designing novel drug delivery vehicles or particles that can be used for cleanup and remediation of environmental contaminants or toxic waste.

\section{Experimental Section}

Materials: PAAm-co-AA $\left(M_{\mathrm{W}}=200000 \mathrm{~g} \mathrm{~mol}^{-1}, 10 \%\right.$ carboxyl) was obtained from Polysciences, Inc. PAA $\left(M_{\mathrm{W}}=250000 \mathrm{~g} \mathrm{~mol}^{-1}\right.$, 35 wt\% solution in water), FITC-dextran $\left(M_{W}=250000 \mathrm{~g} \mathrm{~mol}^{-1}\right)$ and TRITC-dextran $\left(M_{W}=155000 \mathrm{~g} \mathrm{~mol}^{-1}\right)$ were purchased from Sigma-Aldrich. All chemicals were used as received. De-ionized water was used for preparation of the solutions.

Polymer solutions used for co-jetting: Polymer solutions used for co-jetting were made of PAA $(13.1 \% \mathrm{w} / \mathrm{v})$, PAAm-co-AA $(0.044 \% \mathrm{w} / \mathrm{v}), \mathrm{NaOH}(2.19 \% \mathrm{w} / \mathrm{v})$, and FITC-dextran $(0.4 \% \mathrm{w} / \mathrm{v})$ (together solution [a]), PAAm-co-AA ( $5 \% \mathrm{w} / \mathrm{v})$, and PAA $(0.9 \% \mathrm{w} / \mathrm{v})$, TRITC-dextran $(0.4 \% \mathrm{w} / \mathrm{v})$ (together solution [b]) and PAAm-co-AA $(5 \% \mathrm{w} / \mathrm{v})$, PAA $(0.9 \% \mathrm{w} / \mathrm{v})$, and FITC-dextran $(0.4 \% \mathrm{w} / \mathrm{v})$ (together solution $\left.\left[\mathrm{a}^{\prime}\right]\right)$.

Electrohydrodynamic co-jetting with side-by-side dual capillaries: The solutions were separately charged into $1 \mathrm{~mL}$ syringes. A dual-channel tip (FibriJet, Micromedics, Minnesota, USA) with two capillaries (26 gauge, length of 4 inch) was connected to the syringes and placed $33 \mathrm{~cm}$ above a surface of aluminum foil. In order to obtain stable polymer jetting, a flow rate of $100 \mu \mathrm{Lh}^{-1}$ and a voltage difference of $16-17 \mathrm{kV}$ between the capillaries and the collecting surface were applied.

Crosslinking reaction: The crosslinking reaction between amine and carboxylic groups present in the particles was performed by incubating the particles at $170{ }^{\circ} \mathrm{C}$ for $2 \mathrm{~h} .{ }^{[37]}$

Confocal microscopy: Confocal microscopy images were obtained using a SP2 confocal laser scanning microscope (Leica, USA). FITC and TRITC were excited by an Ar-ArKr $(488 \mathrm{~nm})$ and an GreNe $(543 \mathrm{~nm})$ laser, respectively. Fluorescence emissions were collected in the range of 508-523 nm for FITC and 568-583 nm for TRITC.

Scanning electron microscopy (SEM): Samples were used without further coating with a conductive layer for SEM imaging using a FEl quanta 200 3D environmental scanning electron microscope.

Average size distribution calculations: Average diameters of the particles in water, acid, or base solutions were determined based on confocal microscopy images by measuring diameters of more than 100 particles and calculating a mean value.

Molecule uptake studies: 2-aminoacridone ( $\geq 98 \%$, HPLC) (AAC, 3-acetyl-umbelliferon ( $\geq 98 \%$, HPLC) (AU), 6-carboxyfluorescein ( $\geq 95 \%$, HPLC) (CFI), Eosin yellowish solution (EYS), rhodamine $B(R B)$, rhodamine $B$ base (RBB) were purchased from Sigma-Aldrich. Solutions of AAC $(0.0006 \% \mathrm{w} / \mathrm{v})$, AU $(0.00004 \%$ $w / v)$, CFI $(0.0004 \% w / v)$, EYS $(0.0001 \% w / v)$, RB $(0.00004 \% w / v)$ and $\operatorname{RBB}(0.00013 \% \mathrm{w} / \mathrm{v})$ were prepared in deionized water, and solutions of dye pairs were prepared with a $1: 1$ ( $\mathrm{v}: \mathrm{v}$ ratio). To examine the selective absorption behavior of the core/shell particles, the particles were incubated in solutions of dye pairs for $3 \mathrm{~h}$, and then separated from the solutions by centrifugation at 12000 RPM for $10 \mathrm{~min}$. 


\section{Acknowledgements}

We thank Prof. M. Solomon for use of the confocal laser scanning microscope and Dr. M. Yoshida for insightful comments on the manuscript.

[1] H. Ichikawa, Y. Fukumori, J. Controlled Release 2000, 63, 107.

[2] X. P. Qiu, S. Leporatti, E. Donath, H. Möhwald, Langmuir 2001, 17, 5375.

[3] V. C. Lopez, S. L. Raghavan, M. J. Snowden, React. Funct. Polym. 2004, 58, 175.

[4] T. L. U. Nguyen, B. Farrugia, T. P. Davis, C. Barner Kowollik, M. H. Stenzel, J. Polym Sci, Part A: Gen. Pap. 2007, 45, 3256.

[5] Y. Y. Liu, H. Miyoshi, M. Nakamura, Colloids Surf, B 2007, 58, 180.

[6] C. Nayral, E. Viala, P. Fau, F. Senocq, J. C. Jumas, A. Maisonnat, B. Chaudret, Chem. -Eur. J. 2000, 6, 4082.

[7] E. A. Guliants, R. Schwarb, H. Bearbower, J. R. Gord, C. E. Bunker, Rev. Adv. Mater. Sci. 2005, 10, 289.

[8] O. P. Tiourina, A. A. Antipov, G. B. Sukhorukov, N. L. Larionova, Y. Lvov, H. Möhwald, Macromol. Biosci. 2001, 1, 209.

[9] D. Ma, Z. J. Jakubek, B. Simard, J. Nanosci. Nanotechnol. 2006, 6, 3677.

[10] C. Strandberg, A. C. Albertsson, J. Polym. Sci, Part A: Gen. Pap. 2006, 44, 1660.

[11] C. D. Jones, L. A. Lyon, Macromolecules 2000, 33, 8301.

[12] J. H. Kim, T. R. Lee, Chem. Mater. 2004, 16, 3647.

[13] Y. Nakamira, H. Tabata, H. Suzuki, K. Iko, M. Okubo, T. Matsumoto, J. Appl. Polym. Sci. 1986, 32, 4865.

[14] W. D. Hergeth, U. J. Steinau, H. J. Bittrich, H. Tanneberger, Colloid Polym. Sci. 1990, 268, 991.

[15] T. Basinska, S. Slomkowski, M. Delamar, J. Bioact. Compat. Polym. 1993, 8, 205.

[16] C. J. Ferguson, G. T. Russell, R. G. Gilbert, Polymer 2002, 43, 4557.

[17] F. Caruso, R. A. Caruso, H. Möhwald, Science 1998, 282, 1111.

[18] F. Caruso, H. Möhwald, J. Am. Chem. Soc. 1999, 121, 6039.
[19] C. Gao, E. Donath, H. Möhwald, C. Shen, Angew. Chem, Int. Ed. 2002, 41, 3789.

[20] C. Déjugnat, G. B. Sukhorukov, Langmuir 2004, 20, 7265.

[21] S. R. Carter, S. Rimmer, Adv. Funct. Mater. 2004, 14, 553.

[22] Y. W. Zhang, Z. X. Wang, Y. S. Wang, H. X. Zhao HX, C. X. Wu, Polymer 2007, 48, 5639 .

[23] X. L. Luo, A. J. Killard, A. Morrin, M. R. Smyth, Chem. Commun. 2007,3207

[24] S. Liu, J. V. M. Weaver, Y. Tang, N. C. Billingham, S. P. Armes, K. Tribe, Macromolecules 2002, 35, 6121.

[25] S. Fujii, Y. Cai, J. V. M. Weaver, S. P. Armes, J. Am. Chem. Soc. 2005, 127, 7304.

[26] P. Zheng, X. Jiang, X. Zhang, W. Zhang, L. Shi, Langmuir 2006, 22, 9393.

[27] Y. Zhang, M. Jiang, J. Zhao, Z. Wang, H. Dou, D. Chen, Langmuir 2005, 21, 1531.

[28] G. Loscertales, A. Barrero, I. Guerrero, R. Cortijo, M. Marques, A. M. Ganan-Calvo, Science 2002, 295, 1695.

[29] I. G. Loscertales, A. Barrero, M. Marquez, R. Spretz, R. VelardeOrtiz, G. Larsen, J. Am. Chem. Soc 2004, 126, 5376.

[30] Z. Sun, E. Zussman, A. L. Yarin, J. H. Wendorff, A. Greiner, Adv. Mater. 2003, 15, 1929.

[31] J. H. Yu, S. V. Fridrikh, G. C. Rutledge, Adv. Mater. 2004, 16, 1562

[32] Y. Z. Zhang, Z. Huang, X. Xu, C. T. Lim, S. Ramakrishna, Chem. Mater. 2004, 16, 3406.

[33] D. Li, A. Babel, S. A. Jenekhe, Y. Xia, Adv. Mater. 2004, 16, 2062.

[34] Y. Dror, W. Salalha, R. Avrahami, E. Zussman, A. L. Yarin, R. Dersch, A. Greiner, J. H. Wendorff, Small 2007, 3, 1064.

[35] K. H. Roh, D. C. Martin, J. Lahann, Nat. Mater. 2005, 4, 759.

[36] K. H. Roh, D. C. Martin, J. Lahann, J. Am. Chem. Soc. 2006, 128, 6796.

[37] K. H. Roh, M. Yoshida, J. Lahann, Langmuir 2007, 23, 5683.

[38] M. Yoshida, K. H. Roh, J. Lahann, Biomaterials 2007, 28, 2446.

[39] Y. Okahata, H. Noguchi, T. Seki, Macromolecules 1987, $20,15$.

[40] E. Kokufuta, N. Shimizu, I. Nakamura, Biotechnol. Bioeng. 1988, 32, 289.

Received: April 21, 2008 Revised: June 12, 2008 